\title{
Three-dimensional structure of a sunspot light bridge ${ }^{\star}$
}

\author{
T. Felipe ${ }^{1,2}$, M. Collados ${ }^{1,2}$, E. Khomenko ${ }^{1,2}$, C. Kuckein $^{3}$, A. Asensio Ramos ${ }^{1,2}$, H. Balthasar ${ }^{3}$, T. Berkefeld ${ }^{4}$,
} C. Denker ${ }^{3}$, A. Feller ${ }^{5}$, M. Franz ${ }^{4}$, A. Hofmann ${ }^{3}$, J. Joshi ${ }^{5}, 6$, C. Kiess ${ }^{4}$, A. Lagg ${ }^{5}$, H. Nicklas ${ }^{7}$, D. Orozco Suárez ${ }^{8}$, A. Pastor Yabar ${ }^{1,2}$, R. Rezaei ${ }^{1,2}$, R. Schlichenmaier ${ }^{4}$, D. Schmidt ${ }^{9}$, W. Schmidt ${ }^{4}$, M. Sigwarth ${ }^{4}$, M. Sobotka ${ }^{10}$, S. K. Solanki ${ }^{5,11}$, D. Soltau ${ }^{4}$, J. Staude ${ }^{3}$, K. G. Strassmeier ${ }^{3}$, R. Volkmer ${ }^{4}$, O. von der Lühe ${ }^{4}$, and T. Waldmann ${ }^{4}$

${ }^{1}$ Instituto de Astrofísica de Canarias, 38205 C/ Vía Láctea, s/n, La Laguna, Tenerife, Spain e-mail: tobias@iac.es

2 Departamento de Astrofísica, Universidad de La Laguna, 38205 La Laguna, Tenerife, Spain

3 Leibniz-Institut für Astrophysik Potsdam (AIP), An der Sternwarte 16, 14482 Potsdam, Germany

${ }^{4}$ Kiepenheuer-Institut für Sonnenphysik, Schöneckstr. 6, 79104 Freiburg, Germany

5 Max-Planck-Institut für Sonnensystemforschung, Justus-von-Liebig-Weg 3, 37077 Göttingen, Germany

${ }^{6}$ Institute for Solar Physics, Department of Astronomy, Stockholm University, AlbaNova University Centre, 10691 Stockholm, Sweden

7 Institut für Astrophysik, Georg-August-Universität Göttingen, Friedrich-Hund-Platz 1, 37077 Göttingen, Germany

8 Instituto de Astrofísica de Andalucía (CSIC), Apdo. 3040, 18080 Granada, Spain

9 National Solar Observatory, 3010 Coronal Loop Sunspot, NM 88349, USA

10 Astronomical Institute of the Academy of Sciences, Fričcova 298, 25165 Ondřejov, Czech Republic

11 School of Space Research, Kyung Hee University, Yongin, Gyeonggi Do, 446-701, Republic of Korea

Received 25 August 2016 / Accepted 9 November 2016

\section{ABSTRACT}

\begin{abstract}
Context. Active regions are the most prominent manifestations of solar magnetic fields; their generation and dissipation are fundamental problems in solar physics. Light bridges are commonly present during sunspot decay, but a comprehensive picture of their role in the removal of the photospheric magnetic field is still lacking.

Aims. We study the three-dimensional configuration of a sunspot, and in particular, its light bridge, during one of the last stages of its decay.

Methods. We present the magnetic and thermodynamical stratification inferred from full Stokes inversions of the photospheric Si I $10827 \AA$ and Ca I $10839 \AA$ A lines obtained with the GREGOR Infrared Spectrograph of the GREGOR telescope at the Observatorio del Teide, Tenerife, Spain. The analysis is complemented by a study of continuum images covering the disk passage of the active region, which are provided by the Helioseismic and Magnetic Imager on board the Solar Dynamics Observatory.

Results. The sunspot shows a light bridge with penumbral continuum intensity that separates the central umbra from a smaller umbra. We find that in this region the magnetic field lines form a canopy with lower magnetic field strength in the inner part. The photospheric light bridge is dominated by gas pressure (high- $\beta$ ), as opposed to the surrounding umbra, where the magnetic pressure is higher. A convective flow is observed in the light bridge. This flow is able to bend the magnetic field lines and to produce field reversals. The field lines merge above the light bridge and become as vertical and strong as in the surrounding umbra. We conclude that this occurs because two highly magnetized regions approach each other during the sunspot evolution.
\end{abstract}

Key words. Sun: photosphere - Sun: magnetic fields - sunspots - methods: data analysis - methods: observational - Sun: activity

\section{Introduction}

The determination of the magnetic topology of sunspots has been an important topic in solar physics during the past decades. A global scale picture has emerged, showing that sunspots are formed by a flux tube with a strong and vertical magnetic field at the axis, in the umbral center, which becomes weaker and more horizontal at radial distances farther from the flux tube axis (e.g., Tiwari et al. 2015). The improvement in the resolution of the observations has revealed a more complex picture, where fine structure is present in the umbra and penumbra of sunspots (see Solanki 2003; Borrero \& Ichimoto 2011, for a review). The magnetic structure is a result of the close interplay

\footnotetext{
* Movies associated to Figs. 2 and 13 are available at http://www . aanda.org
}

between plasma motions and magnetic fields, and it is subject to significant changes during the evolution of the sunspot.

The decay of a sunspot is a slow process, which usually takes place over several days. The mechanisms leading to the disappearance of the sunspot's magnetic flux are still not fully understood and remain one of the most challenging problems of sunspot physics. Most sunspots exhibit a large-scale outflow, called moat flow (Meyer et al. 1974), beyond their visible outline, whose physical origin differs from that of the Evershed flow (Löhner-Böttcher \& Schlichenmaier 2013). It was first detected spectroscopically (Sheeley 1972) and then by tracking moving magnetic features (MMFs, Harvey \& Harvey 1973). MMFs with the same polarity as the umbra have been associated with sunspot decay (e.g., Martínez Pillet 2002) because they extract net flux from the spot (Zhang et al. 1992). They disperse this 
flux, which is then removed from the photosphere. However, the flux removal mechanism remains elusive. MMFs move through the moat flow, accumulating at the edge of the moat region. On the other hand, pairs of MMFs with opposite polarities do not contribute to the flux removal since they do not carry net flux. Deng et al. (2007) studied the flow field in a decaying sunspot and concluded that the mechanisms contributing to the decay are the fragmentation of the umbra, cancellation of MMFs that encounter opposite polarity areas at the moat's outer boundary, and flux transport by MMFs to regions with the same polarity as the sunspot. After the disappearance of the sunspot, the flow pattern in that location exhibits significant differences with flows at other quiet-Sun regions (Verma et al. 2012). Bellot Rubio et al. (2008) studied the decay of a penumbra using spectropolarimetric data. Blueshifted finger-like structures of weak and horizontal magnetic field extending outward from the border of the umbra were identified. The authors proposed that these structures are penumbral field lines that rise to the chromosphere and contribute to the disappearance of the penumbra at photospheric levels.

Sunspot light bridges can be formed by the fragmentation of the umbra in the decay phase or during the merging of different magnetized areas during the formation of the sunspot in complex active regions (Bray \& Loughhead 1964; Garcia de La Rosa 1987). At the last stages of a sunspot, the photospheric-like conditions are recovered as a consequence of the splitting of the umbra, and a granulation pattern similar to that of the quiet Sun is found in light bridges (Vazquez 1973), although the light-bridge convection cells differ significantly from normal granules (Lagg et al. 2014). Understanding the decay of sunspots requires the study of the magnetic and dynamical structure of light bridges. Previous studies have classified light bridges based on their morphological properties. Light bridges separating two umbral regions are called strong light bridges (e.g., Sobotka et al. 1993, 1994; Jurčák et al. 2006; Rimmele 2008). Their brightness is similar to that of the penumbra, and they typically appear between two regions with the same polarity. Faint light bridges (e.g., Lites et al. 1991; Sobotka et al. 1993) are elongated bright structures that penetrate the umbra. They are composed of rows of bright grains with sizes comparable to umbral dots. Light bridges can exhibit different internal structures. Most of them show several segments along their length that resemble granules (Berger \& Berdyugina 2003), while some light bridges are similar to the bright filaments seen in the penumbra (Lites et al. 2004). All types show a weakened magnetic field strength relative to the surrounding umbra and more strongly inclined field lines (e.g. Beckers \& Schröter 1969; Lites et al. 1991; Leka 1997), which in some case even exhibit a polarity reversal (Lagg et al. 2014). Observations are consistent with a reduced field strength in the photosphere, with a magnetic canopy extending from either side of the light bridge and merging at the top (Jurčák et al. 2006; Lagg et al. 2014). The velocity field measured in light bridges shows evidence of their convective origin (Rimmele 1997; Hirzberger et al. 2002; Lagg et al. 2014). Recently, Schlichenmaier et al. (2016) have also found evidence that the umbral magnetic field is wrapped around light bridges. The authors defined a new type, the plateau light bridge, with Y-shaped dark canals that resemble penumbral grains and suggest the presence of inclined magnetic fields. Several dynamic phenomena have been detected at the chromosphere above light bridges, including recurrent plasma ejections visible in $\mathrm{H} \alpha$ (Roy 1973; Asai et al. 2001), Ca II H (Shimizu et al. 2009), and other bands (AIA 1600 and $1700 \AA$ A, IRIS 1330 and $1400 \AA$, Toriumi et al. 2015), brightness enhancements in the TRACE $1600 \AA$ band (Berger \& Berdyugina 2003), or fan-shaped jets observed in $\mathrm{H} \alpha$ (Robustini et al. 2016). All these processes are considered to be caused by the interaction of the light bridge magnetic field with the surrounding atmosphere through magnetic reconnection events.

Sunspot evolution is driven by the interaction of magnetic fields and plasma. The relevance of the plasma motions in the molding of the magnetic field is quantified by the plasma- $\beta$ parameter (e.g., Gary 2001). It is defined as the ratio between the gas pressure, $P_{\mathrm{g}}$, and the magnetic pressure, $P_{\mathrm{m}}$, i.e., $\beta=$ $P_{\mathrm{g}} / P_{\mathrm{m}}=2 \mu_{0} P_{\mathrm{g}} / B^{2}$, where $\mu_{0}$ is the magnetic permeability and $B$ is the magnetic field strength. In those regions where $\beta \gg 1$, the plasma dominates the magnetic field, which can be bent and dragged following plasma motions. On the other hand, where $\beta \ll 1$, the magnetic field is not influenced by the plasma, and motions can only occur along field lines. In these regions the magnetic field acquires a force-free configuration (see Sect. 2.4 from Borrero \& Ichimoto 2011). In sunspot atmospheres, the umbra shows low $\beta$ values $(\beta<1)$ at the low photosphere and higher values $(\beta>1)$ towards the edges of the spot (i.e., Mathew et al. 2004), although for at least one other sunspot, plasma $\beta>1$ everywhere in the sunspot's lower atmosphere (Solanki et al. 1993). At higher layers the atmosphere is dominated by the magnetic field $(\beta \ll 1)$.

In this study, we aim at analyzing the thermodynamic and magnetic structure of a sunspot during its decay, a few days before it completely disappeared. We concentrate on the deep photosphere, where the magnetic field configuration is more dependent on the plasma dynamics. In Sect. 2 we present the data set and qualitatively describe the evolution of the active region during its disk passage. Section 3 shows the inversion techniques used in the analysis, and the results are described in Sect. 4. Finally, conclusions are outlined in Sect. 5.

\section{Observations}

Spectropolarimetric data of the active region NOAA 12096 were obtained on 2014 June 27 from 8:28 to 9:13 UT with the 1.5-m GREGOR solar telescope (Schmidt et al. 2012) at the Observatorio del Teide, Tenerife, Spain. The center of the analyzed region was located at the solar position $x=-184^{\prime \prime}, y=$ $100^{\prime \prime}$, which corresponds to a heliocentric angle of $\Theta=12.4^{\circ}$ $(\mu=\cos \Theta=0.98)$.

The full data set consists of 10 maps of the four Stokes parameters, each of them covering a scanned area of $61^{\prime \prime} \times$ $7.8^{\prime \prime}$, acquired with the GREGOR Infrared Spectrograph (GRIS, Collados et al. 2012). The pixel size along the slit was $0.135^{\prime \prime}$. For the construction of the maps, 60 consecutive slit positions were included by shifting the slit in increments of $0.135^{\prime \prime}$. Ten accumulations were acquired per recording, with an integration time of $40 \mathrm{~ms}$ each. A full map was completed in $4.5 \mathrm{~min}$. The wavelength scale was calibrated on an absolute scale (Martínez Pillet et al. 1997), correcting for several systematic effects such as Earth's orbital motions and the solar gravitational redshift (see Appendices A and B of Kuckein et al. 2012b). The spectral range spans from 10823.73 to $10842.01 \AA$ with a spectral sampling of $18.1 \mathrm{~m} \AA ~ p i x e l^{-1}$. Figure 1 shows the Stokes $I$ and $V$ parameters at location $\mathrm{C}$ near the umbra/penumbra boundary (see Fig. 3). The spectral range of the spectropolarimeter comprises several spectral lines of interest, such as the photospheric Si I $10827 \AA$ and Ca I $10839 \AA$ lines and the chromospheric He I $10830 \AA$ triplet. In this study we focus on the analysis of the two aforementioned photospheric lines. 
T. Felipe et al.: Three-dimensional structure of a sunspot light bridge

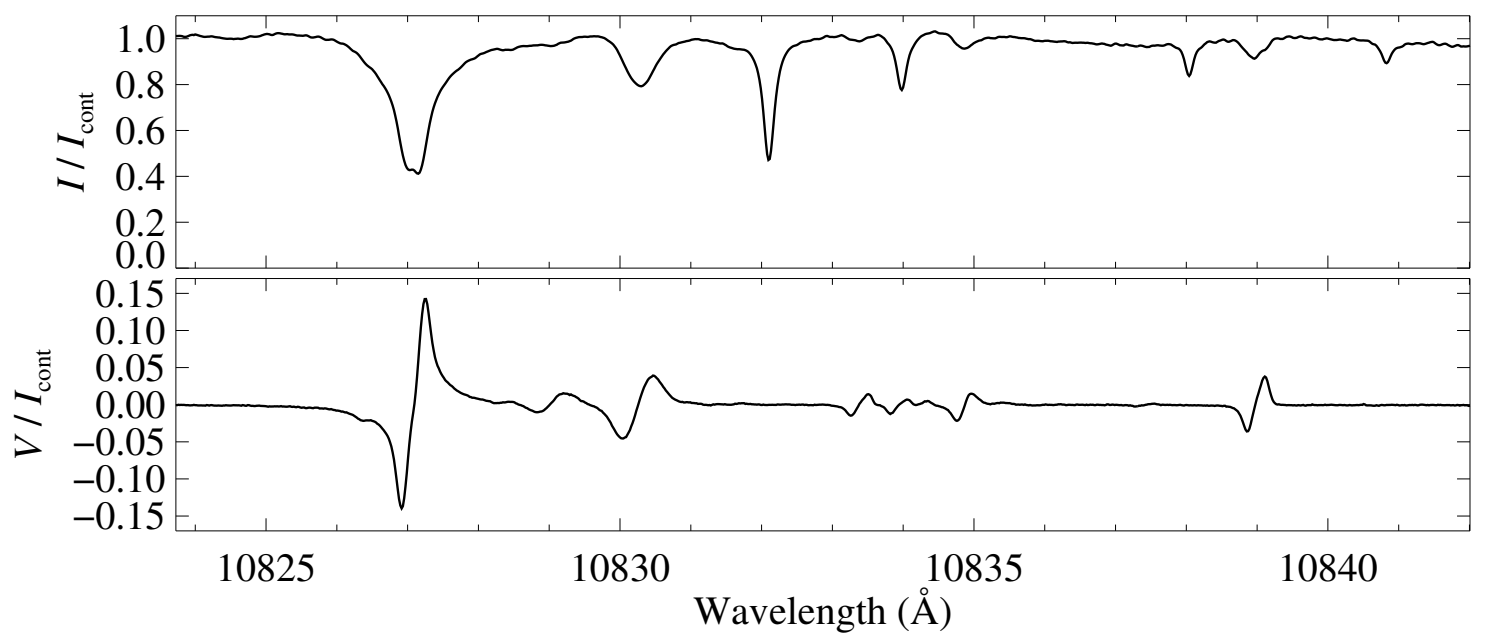

Fig. 1. Stokes I (top panel) and Stokes V (bottom panel) at position C in Fig. 3 normalized to the quiet-Sun continuum intensity.

Standard procedures for dark and flat-field correction and polarimetric calibration (Collados 1999, 2003) were applied to all images. The telescope calibration unit (Hofmann et al. 2012) was used for the polarimetric calibration. The GREGOR Adaptative Optics System (GAOS, Berkefeld et al. 2012) was locked on nearby structures, which substantially improved the image quality. GREGOR's altitude-azimuthal mount introduced an image rotation (Volkmer et al. 2012). Obtaining cospatial maps required the application of some correction to compensate for this rotation, which amounts to $11^{\circ}$ during the whole period of the observations. However, since the maps do not show significant evolution during the observed $45 \mathrm{~min}$, we have decided to focus the analysis on each individual map so that a temporal series of cospatial maps is not necessary. The image rotation during the acquisition of each map (slightly higher than $1^{\circ}$ ) has been neglected.

\subsection{PSF and stray light corrections}

The spatial resolution of solar ground-based observations is limited by the turbulence in the Earth atmosphere and by the optical properties of the telescope (e.g., Martinez Pillet 1992; Chae et al. 1998; Wedemeyer-Böhm 2008). The distortion that these effects produce on the observations is characterized by the point spread function (PSF), whose contributions are commonly approximated by analytical functions. The PSFs are typically dominated by an Airy pattern and, as a consequence of its tails, light observed at the focal plane is contaminated by light coming from distant spatial locations. This spurious light is known as "stray light". Deconvolution techniques are a common approach for correcting this effect. In the case of two-dimensional observations (broad-band imaging or narrow-band spectroscopy), an effective instantaneous PSF can be estimated observationally (e.g., von der Luehe 1993; van Noort et al. 2005). These techniques are not applicable to maps obtained from the scanning of adjacent spatial steps using a slit spectrograph, since at each scan the PSF includes contributions from surrounding regions that are not covered by the one-dimensional slit. Instead, the deconvolution scheme is applied to each monochromatic map using a static PSF (e.g. Beck et al. 2011; Quintero Noda et al. 2015). The correction for stray light using deconvolution techniques has produced observations with unprecedented spatial resolution and has allowed the study of the Sun at finer scales (e.g., Scharmer et al. 2011; Joshi et al. 2011; Henriques 2012).

Schlichenmaier \& Franz (2013) have claimed that the correction for scattered light can produce artifacts on the velocity measurements if the PSF of the stray light is not well characterized. In this work, we have empirically determined the PSF of the GRIS instrument from observations of the Mercury transit in front of the Sun on 2016 May 9. During the observations, weather conditions were not optimum, with some clouds at the level of the observatory. A wavelength-integrated map of Mercury transiting the solar disk was obtained in the $1.56 \mu \mathrm{m}$ region using GRIS polarimetric mode. The scan was composed of 300 consecutive recordings with a $0.135^{\prime \prime}$ step size. The integration time per individual image was $100 \mathrm{~ms}$ and we obtained three accumulations.

A synthetic circular image simulating the undegraded Mercury covering a homogeneous solar surface was created and convolved with a PSF profile given by

$\phi(r)=\alpha G_{1}\left(r, \sigma_{1}\right)+(1-\alpha) G_{2}\left(r, \sigma_{2}\right)$,

where $G_{1}$ and $G_{2}$ are two Gaussians with the rms widths given by $\sigma_{1}$ and $\sigma_{2}$, respectively. The first Gaussian represents the spatial resolution of the telescope (including the seeing), and the second Gaussian accounts for the instrumental stray light. The parameter $\alpha$ is the relative contribution of each Gaussian. The parameters $\alpha, \sigma_{1}$, and $\sigma_{2}$ were obtained from a nonlinear fit of the synthetic convolved circular image to the observed Mercury map.

The fit yields $\alpha=0.584 \pm 0.001, \sigma_{1}=1.385 \pm 0.001^{\prime \prime}$, and $\sigma_{2}=5.030 \pm 0.001^{\prime \prime}$. The FWHM of the first Gaussian $\left(3.26^{\prime \prime}\right)$ is consistent with the angular resolution of the observations, which were seeing-limited to $3.3^{\prime \prime}$. This fact supports the assumption of the PSF proposed in Eq. (1) and allows us to argue confidently that the second Gaussian gives the stray light contamination, with a FWHM of $11.8^{\prime \prime}$ and a relative weight of approximately $42 \%$.

The spectropolarimetric maps of the sunspot were deconvolved using a principal component analysis regularization (Ruiz Cobo \& Asensio Ramos 2013). We used a PSF given by Eq. (1), but substituted $G_{1}\left(r, \sigma_{1}\right)$ by the Airy profile of the GREGOR telescope with the radius of the aperture corresponding to the Fried parameter $r_{0}$. During the scanning of the map, the average value for the whole scan at $10830 \AA$ was $r_{0}=50.7 \mathrm{~cm}$. Owing to the unfortunate seeing conditions during the Mercury 

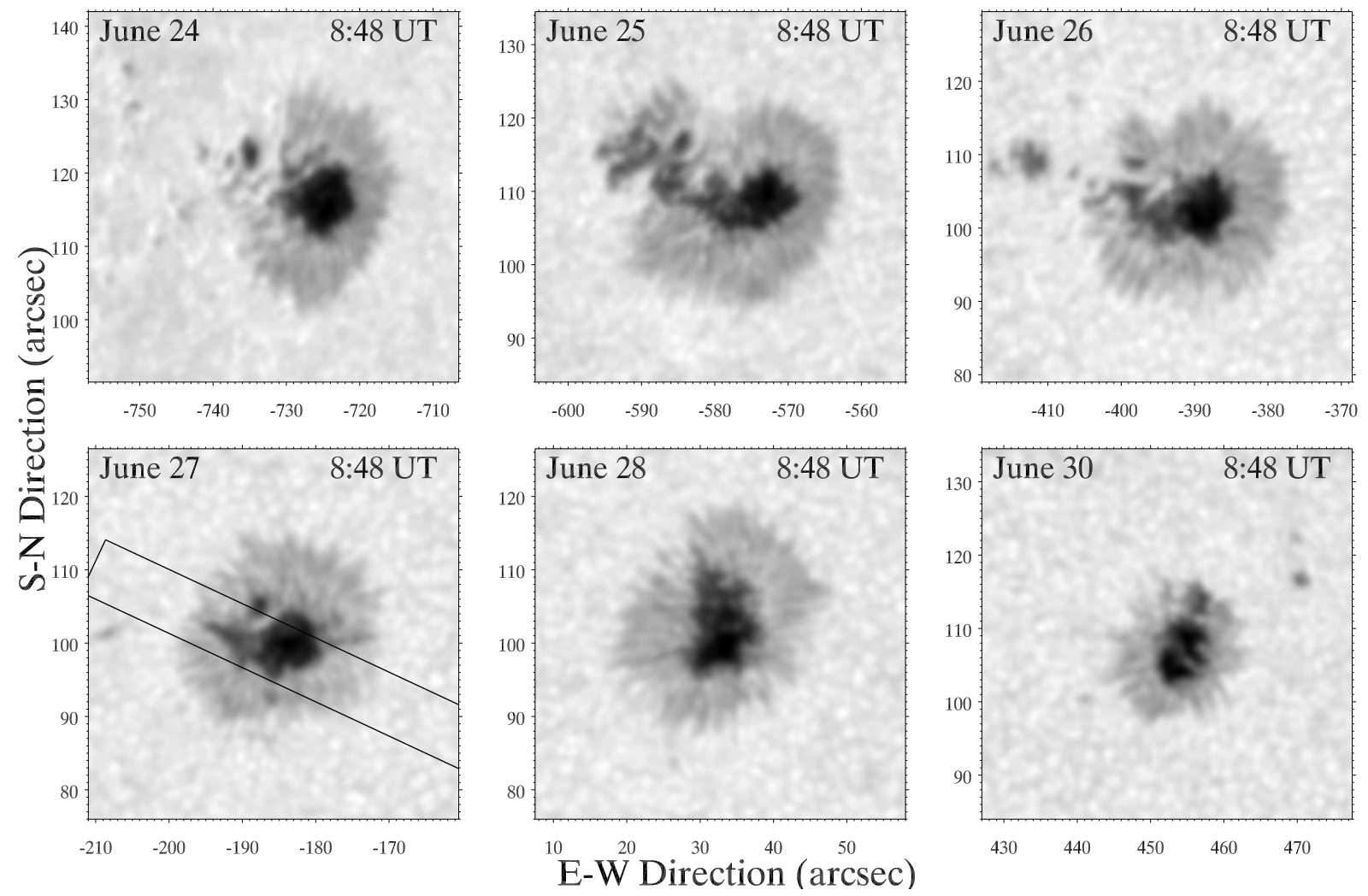

Fig. 2. HMI continuum images of the evolution of the active region NOAA 12096 between 2014 June 24-30. Date and time of the images are indicated at the top of each panel. The tilted rectangle in the bottom left panel indicates the FOV covered by the GRIS observations. The temporal evolution of the sunspot while crossing the solar disk is available as a movie in the online edition. In that movie the location of the sunspot is indicated by a red box in an inset showing the full disk.

transit, the small-scale properties of the stray light can be masked under the seeing contribution to the $G_{1}$ component of the PSF.

\subsection{Evolution of the sunspot}

Continuum images obtained with the Helioseismic and Magnetic Imager (HMI, Schou et al. 2012) on board the Solar Dynamic Observatory (SDO) have been examined to discuss the temporal evolution over a few days of the sunspot NOAA 12096 during its decay. HMI provides full-disk observations with $4096 \times 4096$ pixels and a pixel size of $0.5^{\prime \prime}$. A selection of one image every 12 min between 2014 June 23 UT 16:00 and 2014 July 1 UT 23:48 was extracted from the HMI database. The full temporal series includes 1000 time steps.

Figure 2 shows a sample of six continuum images with a cadence of one day, except for the last panel, which corresponds to two days after the previous snapshot. A movie with the evolution of the sunspot during the time span indicated in the previous paragraph can be found in the online material. Between June 23 and June 26, the sunspot group was classified with a Hale class $\alpha$. During this time, part of the umbra separates from the center of the sunspot and moves toward the east. On June 26 at 08:48 UT, this region is completely detached from the outer penumbra of the sunspot. At the same time, a darker penumbral region (which appears as a dark pore-like structure at $x=-735$, $y=122.5$ on June 24 at 08:48 UT) moves toward the umbra. On June 27, this region is separated from the umbral core and from a spike-like extension of the main umbra by a zone with penumbral brightness. It forms the strong light bridge that is analyzed in this study. Our GRIS observations were taken at that moment. The area covered by them is delineated by solid black lines in the bottom left panel of Fig. 2. At this time, the group was classified as a $\beta$-region, although the region under investigation shows only negative polarity. The dark pore-like feature then merges with the rest of the umbra, forming an extended umbra with internal structure. At the last stages of the evolution of the sunspot, the umbra is divided by a light-bridge into two separated dark regions with the same polarity (see last panel of Fig. 2), and finally it loses the surrounding penumbra. A C-class flare on June 24 at 20:56 UT was associated with this active region.

A section of the field of view (FOV) covered by the GRIS observations is shown in Fig. 3. It corresponds to the continuum intensity at a wavelength close to the Si I $10827 \AA$ line. This is the fifth map of the series acquired with GRIS, and it was obtained between 08:46 and 08:50 UT. Since the ten maps of the series provide similar results, from this point onward all figures and discussion refer to this time step. The contours overplotted on the map delimit the boundaries of some masks that have been applied for the analysis of the data. The region inside the white line includes the darkest part of the umbra, and in the following this mask is referred to as dark umbra. What we term the faint umbra corresponds to the area between the white line and the black solid line and also to the small umbra around $x=13^{\prime \prime}$ and $y=7^{\prime \prime}$. The penumbra is delimited by the black dashed and solid lines. Finally, the area surrounded by the dotted line between the dark pore-like feature and the umbra is called light bridge here.

\section{Spectral line inversion and data analysis}

The photospheric Si I $10827 \AA$ and Ca I $10839 \AA$ lines were inverted simultaneously using the Stokes Inversion based on 


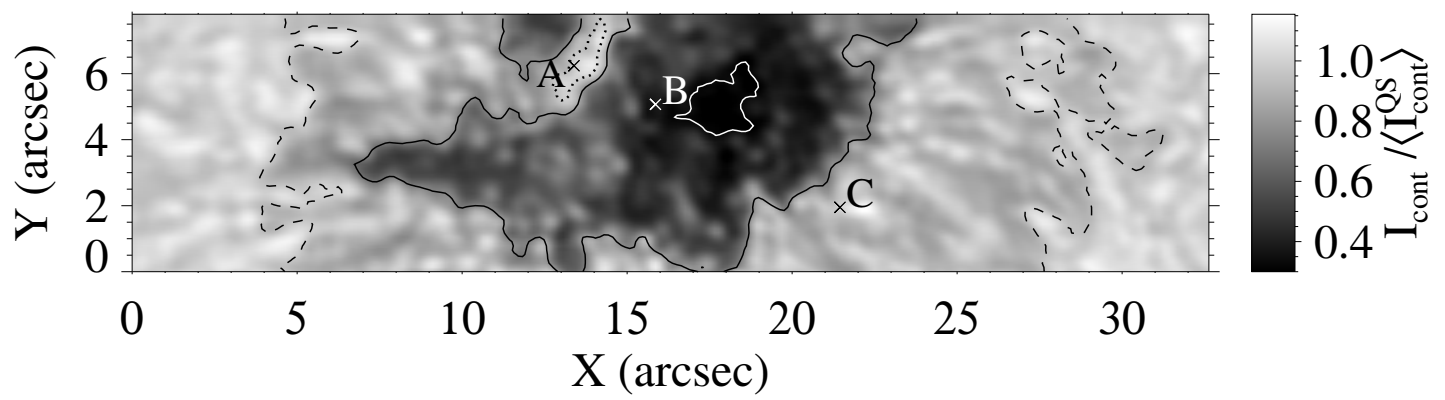

Fig. 3. Continuum intensity map normalized to the average continuum intensity in the quiet Sun $\left\langle I_{\text {cont }}^{\mathrm{QS}}\right\rangle$ obtained with GRIS on June 27 between 08:46 and 08:50 UT. Points $A, B$, and $C$ correspond to the locations used for Figs. 1, 4-6. The contour lines mark the boundaries of the masks used for the analysis in Sect. 4: dark umbra inside the white line, faint umbra delimited by the black solid line and the white line, penumbra between the black solid line and the dashed lines, and the light bridge lies inside the dotted line.

Response functions (SIR) code (Ruiz Cobo \& del Toro Iniesta 1992). SIR yields the thermal, dynamic, and magnetic-depthdependent stratification of the atmosphere that best matches the observed Stokes profiles. The solution is achieved after an iterative process, where the numerical integration of the radiative transfer equation is performed under the assumption of local thermodynamic equilibrium and using the spectral line response functions (RFs), which represent the partial derivatives of the Stokes parameters with respect to the various atmospheric parameters (at different depths).

The Si I $10827 \AA$ A line has been used to probe the photosphere in many solar studies (e.g., Centeno et al. 2006; Bloomfield et al. 2007; Felipe et al. 2010; Kuckein et al. 2012a). Its proximity to the chromospheric He I $10830 \AA$ triplet makes this spectral region an ideal testing ground for simultaneous analyses of the photosphere and chromosphere. On the other hand, the Ca I $10839 \AA$ line, located a few Ångström toward the red, has been widely overlooked (but see Joshi et al. 2016; Balthasar et al. 2016). The broad spectral range of GRIS allows the simultaneous observation of all these spectral lines. The inclusion of the Ca I $10839 \AA$ line in the analysis rewards us with a better sampling of the deeper atmospheric layers, as can be seen in its response functions. Figure 4 shows the response functions of Stokes $I$ to the temperature and Stokes $V$ to the magnetic field strength for the Si I $10827 \AA$ and Ca I $10839 \AA$ lines at location $A$ in Fig. 3. The top panels show that the Si I line is sensitive to heights up to $\log \tau=-3$, while the highest sensitivity of the core of the CaI line is located slightly above the height of the continuum formation. The Stokes $V$ signal of the Si I $10827 \AA$ line is sensitive to the magnetic field in a spectral range of $1 \AA$ centered at the core of the line. The maximum value of the RF at a specific wavelength is found at $\log \tau=-2.7$, where $\tau$ corresponds to the continuum optical depth at $5000 \AA$. On the other hand, the peak of the CaI $10839 \AA$ RF is located at a higher optical depth, around $\log \tau=-0.4$. These results refer to the RFs at location A. We have computed the RFs to magnetic field perturbations at several locations, including quietSun, penumbral, and umbral regions to retrieve an estimate of the average formation height of the Ca I and Si I lines. The analysis shows that their highest sensitivity to the magnetic field is found around $\log \tau=-0.5$ and -2.2 , respectively. We estimate that the RFs of the Ca I $10839 \AA$ line are sufficiently high to retrieve reliable inversions of the data for $\log \tau$ lower than +0.3 . The upper limit, where the atmospheric parameters obtained from the inversions are trustworthy, is given by the RFs of the Si I $10827 \AA$ line. Thus, in the following, all the results
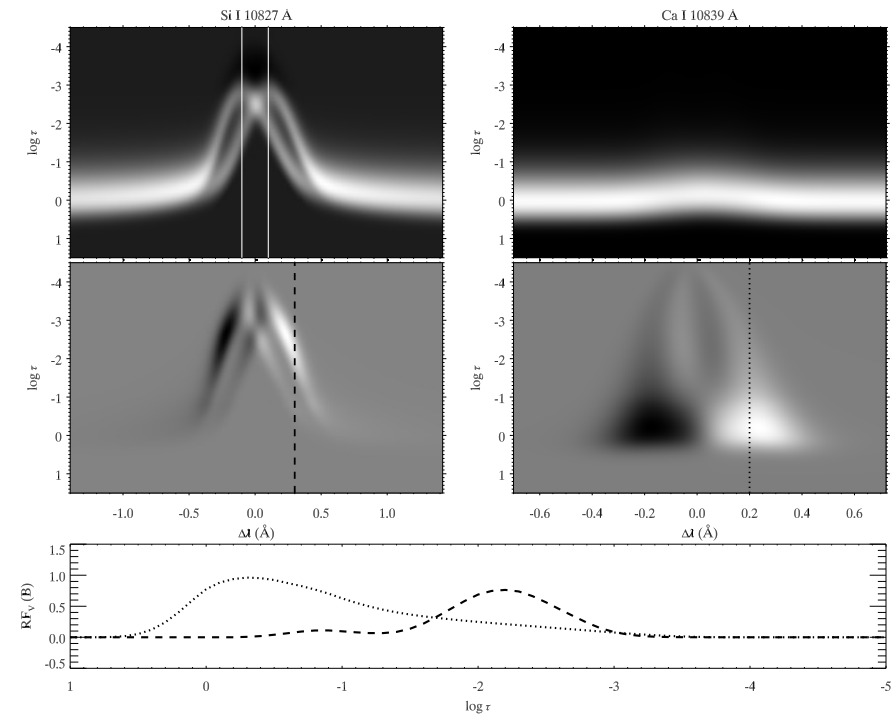

Fig. 4. Normalized response functions of Stokes I to temperature (top panels) and Stokes $V$ to the magnetic field strength (middle and bottom panels) for the atmosphere at location A. Top panels: response functions of Stokes $I$ to temperature of the Si I $10827 \AA$ line (left panel) and CaI $10839 \AA$ line (right panel). The region between the vertical while solid lines has been neglected for the inversions. Middle panels: response functions of Stokes $V$ to magnetic field of the Si I $10827 \AA$ line (left panel) and Ca I $10839 \AA$ line (right panel). Bottom panels: variation of the response functions of Stokes $V$ to the magnetic field of the Si I $10827 \AA$ line (dashed line) and the Ca I $10839 \AA$ line (dotted line) with $\log \tau$ at the wavelengths indicated by the dashed and dotted lines in the middle panels, respectively.

derived from the simultaneous inversions of both lines are restricted to the $\log \tau$ range between +0.3 and -4.0 .

The SIR code requires an initial guess for the atmospheric model. We chose the cool umbral model from Collados et al. (1994), which covers the optical depth range $+1.4 \geq \log \tau \geq-5$.0. The temperature stratification $T(\tau)$ was inverted using five nodes; three nodes were used for the magnetic field strength $B(\tau)$ and the magnetic field inclination $\gamma(\tau)$, and two nodes for the line-of-sight (LOS) velocity $v(\tau)$. The magnetic field azimuth $\chi$ was set to be independent of height, so that a constant azimuth was imposed in the initial model, and only one node was selected for it.

The solar abundances were taken from Asplund et al. (2009), while the atomic data for the Si I $10827 \AA$ line were obtained from Borrero et al. (2003). Since the core of the Si I $10827 \AA$ line is formed in non-LTE conditions, a spectral region with a 

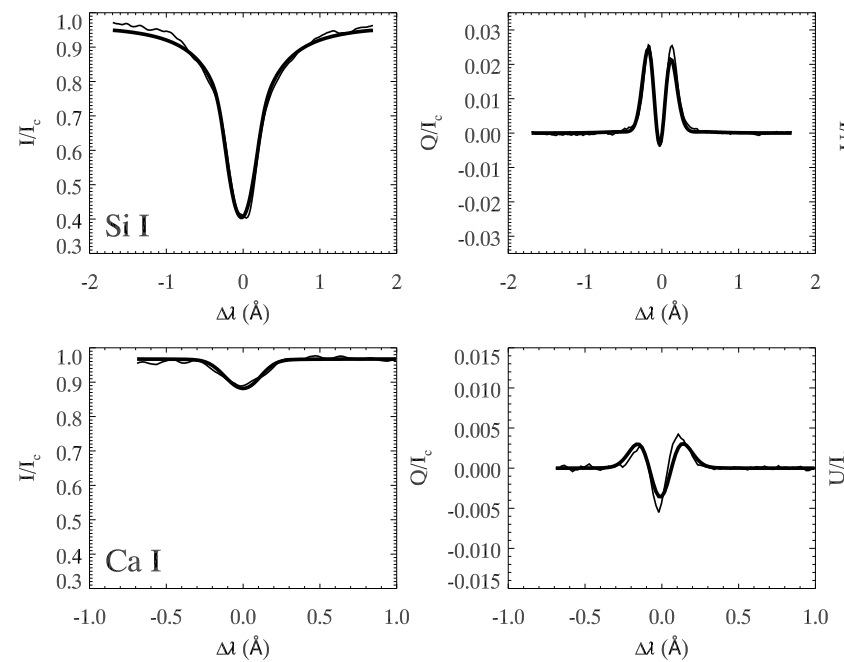
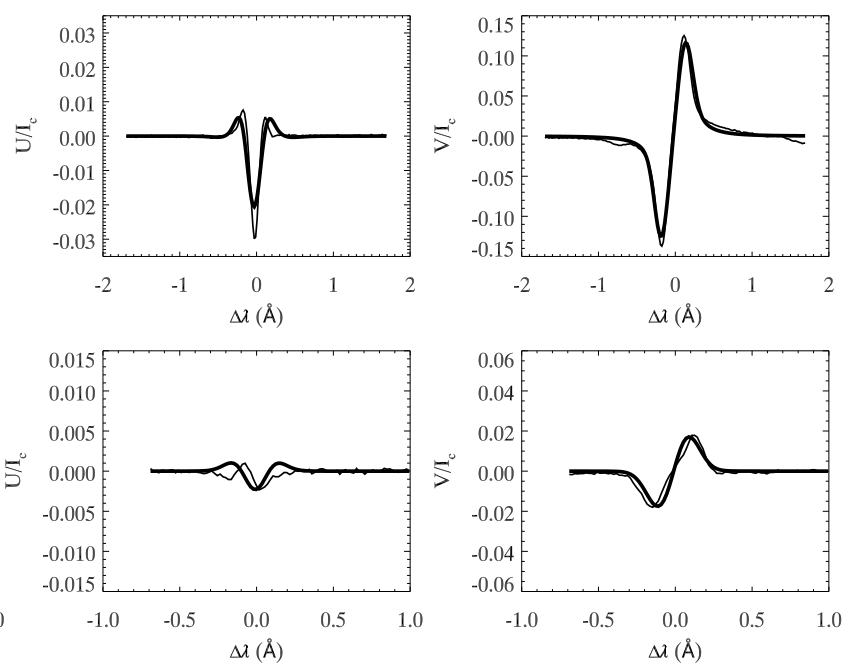

Fig. 5. Stokes parameters at location A (light bridge) in Fig. 3 for the Si I 10827 A line (top panels) and the Ca I $10839 \AA$ line (bottom panel). Thin lines correspond to the observed profiles and thick lines to the fit obtained with SIR. The wavelength $\Delta \lambda=0$ refers to the line core. The physical parameters inferred from the inversion at $\log \tau=-0.5$ are $v=-0.01 \mathrm{~km} \mathrm{~s}^{-1}, B=568 \mathrm{G}, \gamma=107^{\circ}$, and $\chi=240^{\circ}$, and at $\log \tau=-2.2$ are $v=-0.62 \mathrm{~km} \mathrm{~s}^{-1}, B=1339 \mathrm{G}, \gamma=151^{\circ}$, and $\chi=190^{\circ}$.
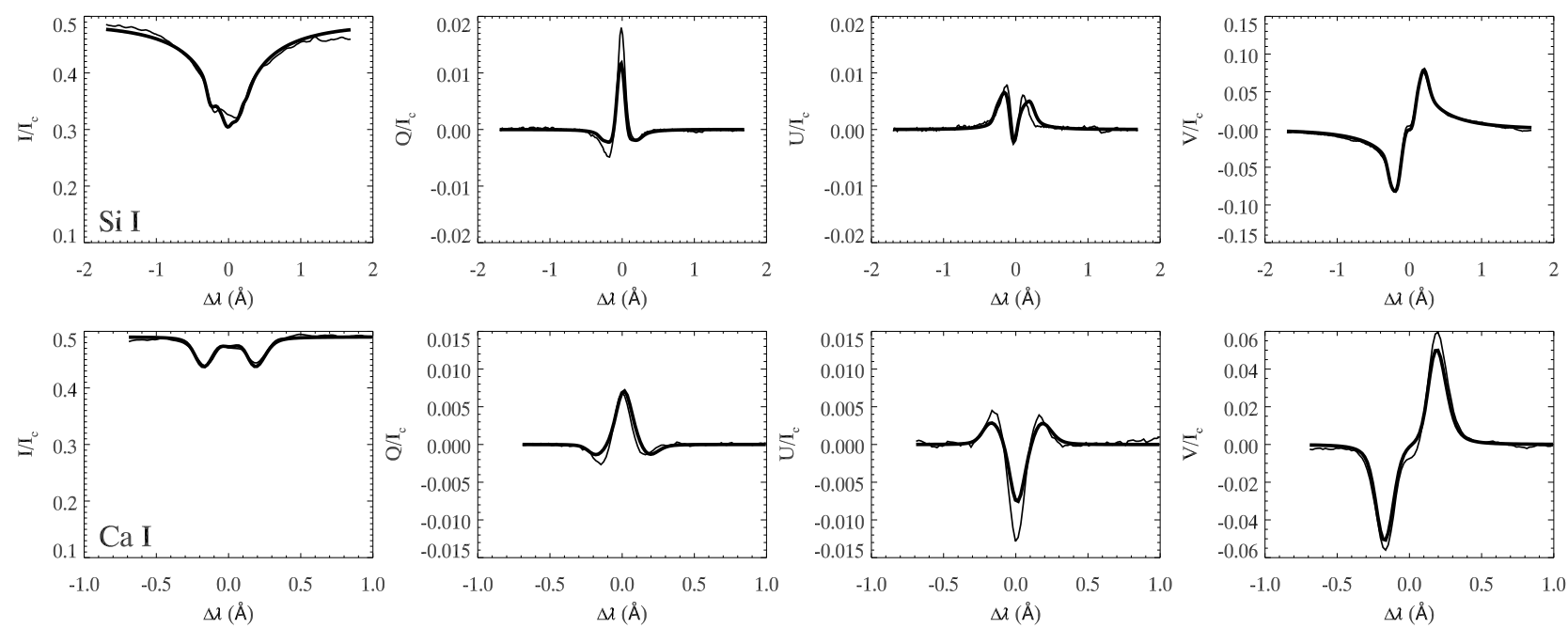

Fig. 6. Same as Fig. 5, but for location $B$ (umbra) in Fig. 3, which corresponds to the faint umbra of the sunspot. The physical parameters inferred from the inversion at $\log \tau=-0.5$ are $v=0.43 \mathrm{~km} \mathrm{~s}^{-1}, B=2203 \mathrm{G}, \gamma=160^{\circ}$, and $\chi=240^{\circ}$, and at $\log \tau=-2.2$ are $v=-0.29 \mathrm{~km} \mathrm{~s}{ }^{-1}$, $B=2224 \mathrm{G}, \gamma=158^{\circ}$, and $\chi=240^{\circ}$.

$0.2 \AA$ width centered on the peak of the line has been neglected for the inversions (see top left panel of Fig. 4). This mask has only been applied to the intensity profile, and the spectral region neglected has been chosen based on a comparison between a quiet-Sun reference atmosphere (HSRA, Gingerich et al. 1971) and the temperature stratification retrieved from inversions of the Si I $10827 \AA$ line from the FTS atlas (Kurucz et al. 1984) with different masks. We have assumed that Stokes $Q, U$, and $V$ are not affected by non-LTE conditions. The atomic parameters of the Ca I $10839 \AA$ line were determined semi-empirically. The line was synthesized iteratively in the HSRA atmosphere by changing the values of the enhancement factor to the van der Waals coefficient $E$ and the logarithm of the multiplicity of the level times the oscillator strength $\log (g f)$. This procedure was repeated until an agreement with the observed intensity in the Ca I $10839 \AA$ line from the FTS atlas was achieved. For the abundances used in this study, we find $E=1.0$ and $\log (g f)=0.10$. The different values of the macroturbulence introduced in the HSRA model used for the synthesis can lead to the determination of different $E$ and $\log (g f)$ parameters. We set the macroturbulence to $1.7 \mathrm{~km} \mathrm{~s}^{-1}$, since with this value and the atomic parameters of the Si I $10827 \AA$ line from Borrero et al. (2003), the intensity profile of the Si I line synthesized in the HSRA model reproduces that from the FTS atlas.

Figures 5 and 6 show a comparison between the observed Stokes profiles (thin line) and the results from the simultaneous inversion of the two photospheric lines (thick line) at two different positions. Each figure is composed of eight panels that represent the Stokes $I, Q, U$, and $V$ parameters for the Si I $10827 \AA$ line (top panels) and the Ca I $10839 \AA$ line (bottom panels). Figure 5 shows the profiles at a point in the light bridge. Its location is indicated with the letter $A$ in Fig. 3. Figure 6 corresponds to the umbral atmosphere marked as $B$ in Fig. 3. In both locations the SIR results show a satisfactory performance, providing an inferred atmosphere that captures the most relevant 


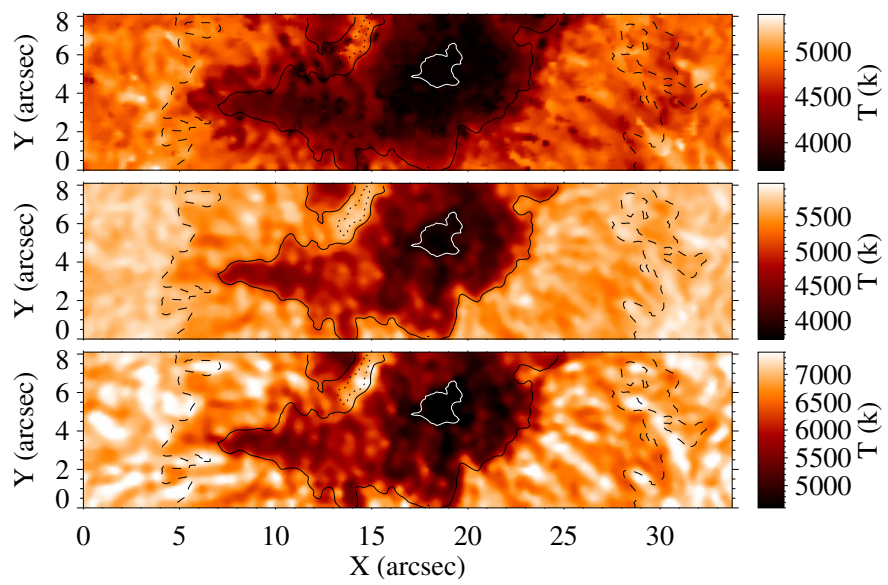

Fig. 7. Temperature map inferred from simultaneous inversions of Ca I $10839 \AA$ and Si I $10827 \AA$ lines at $\log \tau=-2.2$ (top panel), $\log \tau=-0.5$ (middle panel), and $\log \tau=0.3$ (bottom panel). Contour lines correspond to the same masks as defined in Fig. 3.

features present in the observed profiles. The continuum next to the Si I $10827 \AA$ line is slightly below that from the observed profiles. Deep-layer temperatures depending on this line might be a bit low. At the umbral position (Fig. 6), the intensity absorption profile of the Ca I $10839 \AA$ line is decomposed into two $\sigma$-components with circular polarization, as can be seen in the Stokes $V$ profile. This fact points to a strong magnetic field in the direction of the LOS. According to the sign of the lobes in Stokes $V$, the polarity of the umbra is negative. On the other hand, at location A the magnetic field is not strong enough to produce a visible Zeeman splitting in the intensity of the Ca I $10839 \AA$ line. The reduction of the LOS magnetic field is also evident in the reduced wavelength shift of the Stokes $V$ lobes. Stokes $Q$ and $U$ show significant values, indicating a considerable horizontal magnetic field.

The determination of the magnetic field perpendicular to the LOS from the linear polarization signals (Stokes $Q$ and $U$ ) leads to two compatible solutions that differ by $180^{\circ}$ in its azimuthal direction. Several techniques have been developed to resolve this ambiguity (e.g., Lites et al. 1995; Leka et al. 2009). We have addressed this issue by assuming an approximately radial structure inside the sunspot and carefully chose the azimuth origin around the center of the spot. The azimuth value retrieved from the inversion was changed by $180^{\circ}$ in those regions where it was needed to satisfy this assumption.

\section{Results}

The inversion procedures were applied to every spatial position within the FOV of GRIS observations. A median filter was applied to the inversion results to remove the spurious values of the atmospheric parameters retrieved at those individual pixels where the convergence of the inversion is inadequate (at the locations where the differences between the observed and synthetic Stokes spectra resulting from the inversion, quantified by the $\chi^{2}$, are too high). The value of each parameter at a certain optical depth and spatial coordinates $X$ and $Y$ was substituted by the median in a $3 \times 3$ pixel neighborhood.

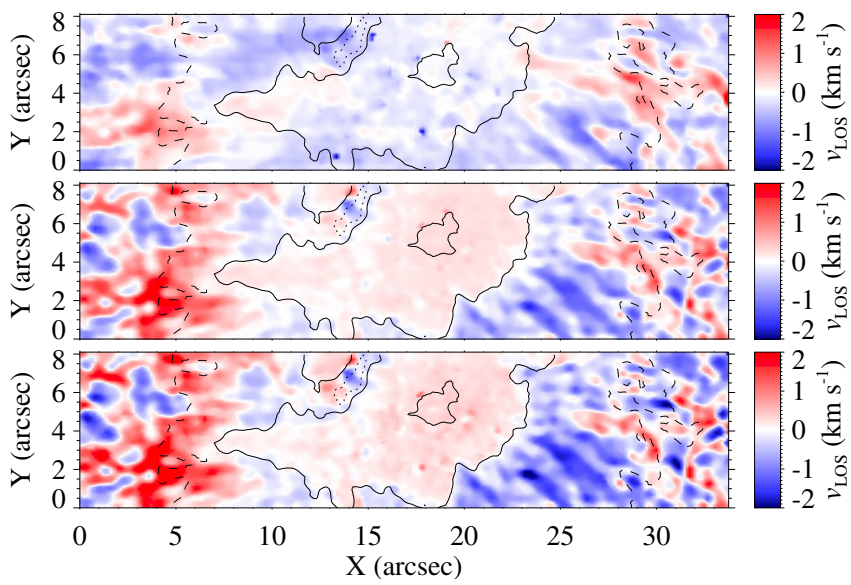

Fig. 8. Same as Fig. 7, but for the LOS velocity. The color scale is saturated between -2 and $2 \mathrm{~km} \mathrm{~s}^{-1}$.

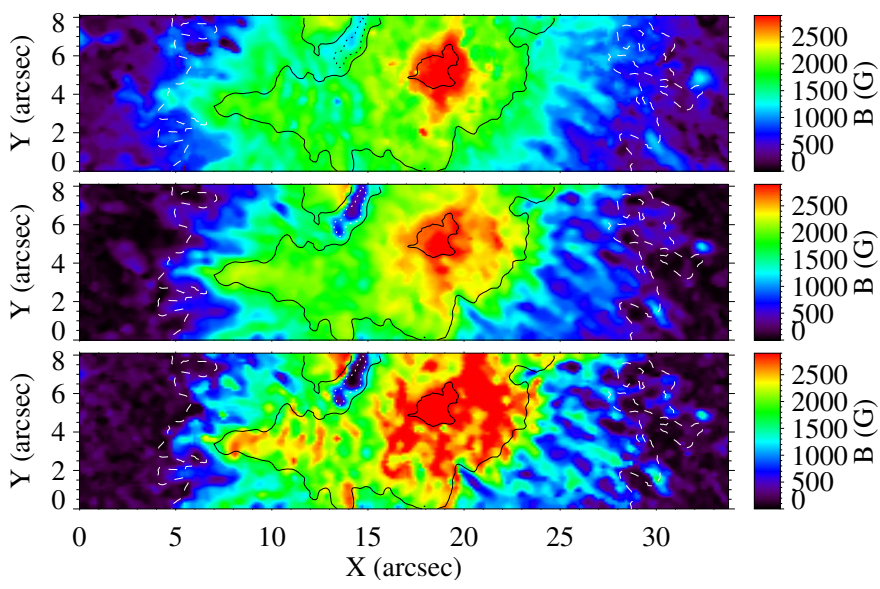

Fig. 9. Same as Fig. 7, but for the magnetic field strength.

\subsection{Examination of the atmosphere}

Figures 7-10 show maps of temperature, LOS velocity, and magnetic field strength and orientation for the same region and time as illustrated in Fig. 3. The contour lines indicate the masks defined in Fig. 3. Maps at three different atmospheric levels, corresponding to $\log \tau=0.3,-0.5$, and -2.2 are plotted. As pointed out in Sect. 3, the two higher layers correspond to the peaks in sensitivity of the $\mathrm{Ca}$ I and $\mathrm{Si}$ I lines to the magnetic field, while $\log \tau=0.3$ is the deepest layer where we consider that our inversion results are trustworthy.

The general structure of the sunspot is consistent with a vast number of earlier studies (e.g. Lites et al. 1993; del Toro Iniesta et al. 1994; Stanchfield et al. 1997; Westendorp Plaza et al. 2001; Bello González et al. 2005; Langhans et al. 2005; Balthasar \& Collados 2005; Bellot Rubio et al. 2007; Beck 2008; Balthasar \& Gömöry 2008; Tiwari et al. 2015). The top panel of Fig. 11 shows the variation with optical depth of the temperature averaged over the regions delimited by the masks defined in Fig. 3. At lower atmospheric heights there is a stark contrast between the temperature of dark umbra, faint umbra, and the other regions. Below $\log \tau=-0.6$ the light bridge shows quiet-Sun temperatures, and for $\log \tau$ between -0.6 and -2 its temperature is even higher than that of the quiet Sun. At the higher atmosphere the light bridge temperature exhibits a steep gradient, reaching an umbral value around $\log \tau=-3$. 


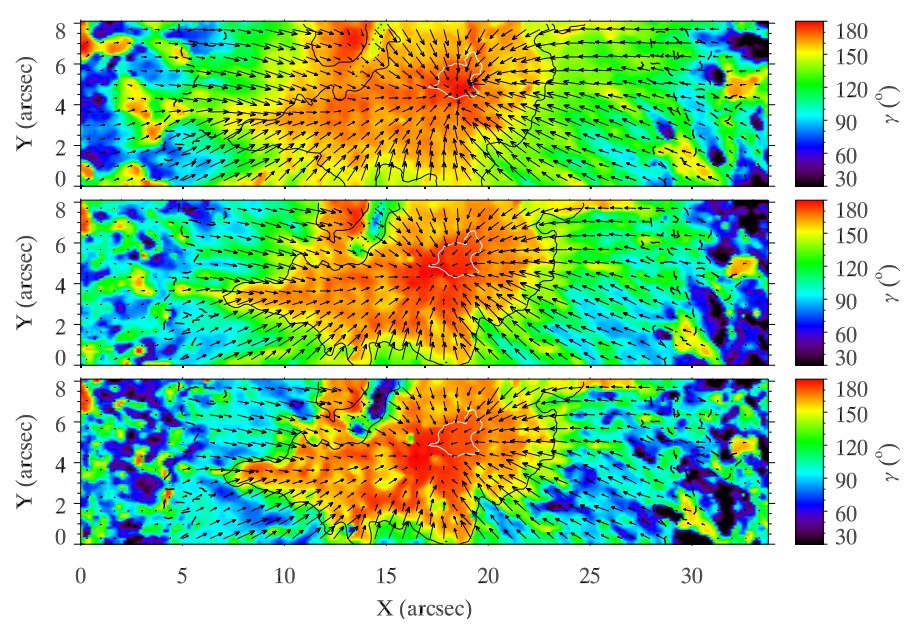

Fig. 10. Same as Fig. 7, but for the magnetic field inclination in the local solar reference frame. Black arrows indicate the direction of the horizontal magnetic field.

The two deeper velocity maps from Fig. 8 clearly show the Evershed flow (Evershed 1909). Interestingly, at $\log \tau=0.3$ the light bridge shows an upflow with a velocity of $-1 \mathrm{~km} \mathrm{~s}^{-1}$ surrounded by two downflows, the strongest with a velocity of $2 \mathrm{~km} \mathrm{~s}^{-1}$. These flows are present only at the side of the light bridge next to the small umbra. The downflows decrease with height and completely vanish by $\log \tau=-2.2$ (top panel). The upflow maintains its value of around $-1 \mathrm{~km} \mathrm{~s}^{-1}$ for all the optical depths we analyzed.

The atmospheres obtained from the inversion are referred to a system of reference with the vertical directed along the LOS. The position of the active region on the solar disk means that this reference frame does not coincide with the local reference frame, where vertical magnetic fields are radially oriented. The magnetic field has been transformed to the solar local reference frame. The strength and orientation of the magnetic field are illustrated in Figs. 9 and 10. At the three plotted optical depths, the photospheric magnetic field ranges from $3000 \mathrm{G}$ strength and a nearly vertical orientation at the center of the umbra to $1000 \mathrm{G}$ and more horizontal inclination at the penumbra. At the deepest layer $(\log \tau=0.3)$ the inclination of the penumbral magnetic field is higher, showing values around $90^{\circ}$ and even reversals.

At the lower atmosphere the light bridge shows a strong reduction in magnetic field strength, with the lowest trustworthy value as low as $30 \mathrm{G}$, and becomes more horizontal. At some locations this low magnetic field results in a polarity reversal. The light bridge average field inclination at the deepest layer is $76^{\circ}$, but at certain locations its inclination is around $25^{\circ}$. Figure 11 shows that for heights below $\log \tau=-0.5$ the magnetic field in the light bridge is more horizontal and has lower strength than the penumbral magnetic field. At higher levels its strength increases to above that of the penumbral average, and at the same time it becomes more vertical. The configuration of the magnetic field in the light bridge around $\log \tau=-2.2$ is closer to the faint umbra than the penumbra, according to its inclination of around $150^{\circ}$ and its higher strength. The topology of the magnetic field in the light bridge can be pictured as a region with a low horizontal magnetic field (including strong reversals in the magnetic field lines) that is covered by a canopy of stronger and more vertical field, similar to the surrounding umbral field (Jurčák et al. 2006; Lagg et al. 2014; Schlichenmaier et al. 2016).

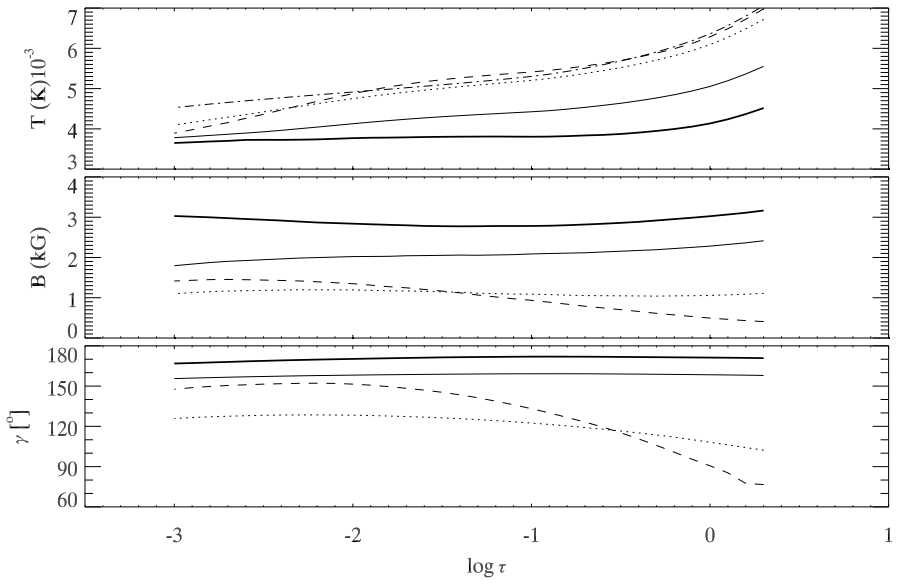

Fig. 11. Stratification of the atmospheric parameters averaged over the region delimited by the masks defined in Fig. 3. From top to bottom: temperature, magnetic field strength, and LOS magnetic field inclination. Thick solid line: dark umbra; thin solid line: faint umbra, dotted line: penumbra; dashed line: light bridge; dashed-dotted line: quiet Sun (only in the top panel).

\subsection{Geometrical scale}

The optical depth scale $(\log \tau)$ retrieved with SIR was converted into a geometrical scale $(z)$ assuming hydrostatic equilibrium. At each location, the hydrostatic equilibrium was re-evaluated taking into account magnetic pressure, but neglecting magnetic tension. Next, the geometrical height was obtained from the new density and the opacity evaluated at a continuum wavelength. The Wilson depression causes a change in the reference height of the geometrical scale for each position. We chose to define $z=0$ at the height of $\log \tau=0$ in the quiet-Sun atmosphere. All the inverted atmospheres were shifted in the vertical direction to determine the configuration that satisfies the pressure balance. In the sunspot, this is given by (e.g., Mathew et al. 2004)

$P_{0}(z)=P_{\mathrm{g}}(r, z)+B_{\mathrm{z}}^{2}(r, z) /\left(2 \mu_{0}\right)$,

where $P_{0}(z)$ is the gas pressure stratification in the quiet Sun, $P_{g}(r, z)$ is the gas pressure in the spot, $B_{z}(r, z)$ is the vertical magnetic field, and $\mu_{0}$ is the magnetic permeability. The curvature force was neglected, similarly to previous works (e.g., Sánchez Almeida 2005; Mathew et al. 2004). For a discussion of the influence of magnetic tension on the estimation of the Wilson depression, see Martinez Pillet \& Vazquez (1993) and Solanki et al. (1993). We determined $P_{0}(z)$ as the average of the gas pressure throughout the quiet Sun surrounding the sunspot. The Wilson depression $\Delta z_{\mathrm{WD}}(x, y)$ is determined as the shift in $z$ that needs to be applied to the total pressure (gas and magnetic pressure) at every position $[x, y]$ to match $P_{0}$ at $\log \tau=0$ (which we set as $z=0$ in the quiet Sun). This method proved to be problematic when determining $\Delta z_{\mathrm{WD}}(x, y)$ in the umbra and regions with high magnetic field strength. In those cases, for all the heights where the response functions of the spectral lines are large enough, the total pressure in the magnetized atmosphere was higher than $P_{0}(z=0)$. This might be partially caused by an inaccurate gas pressure retrieved from the inversion in the magnetized regions, since the inversion code does not include the magnetic pressure term in the derivation of the pressure scale. We determined the change in the Wilson depression of every position with respect to an arbitrarily chosen location with a moderate field strength, instead of the average quiet Sun. In the quiet-Sun atmosphere, the range of heights where the inversion provides a reliable result spans around $450 \mathrm{~km}$, while typical Wilson depressions can 


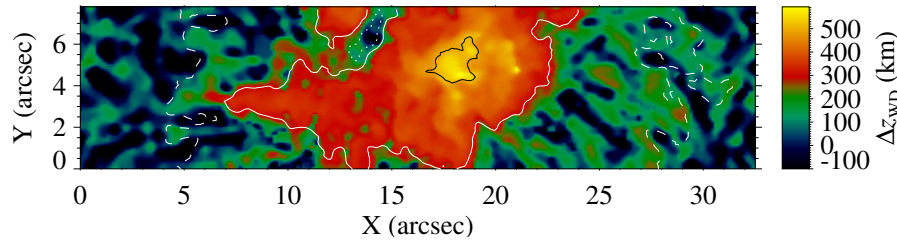

Fig. 12. Wilson depression of the observed sunspot. Contour lines correspond to the masks defined in Fig. 3.

be higher than $600 \mathrm{~km}$. This means that in some umbral regions the vertical displacement required for matching the photospheric pressures is higher than the available atmosphere. The total pressure stratification of the reference atmosphere is between the low (quiet Sun) and strong (umbra) field cases, and the geometrical shift can be directly obtained at all locations. The absolute Wilson depression was then retrieved by adding the shift between the average quiet Sun and the reference position.

Our estimation of the Wilson depression has some limitations. First, we neglected the magnetic tension. A rigorous determination would require solving the magnetohydrodynamic equations on a three-dimensional grid. The motion equation could be solved even including the velocity and its gradients, although in this case we would miss information about the horizontal components and the temporal derivatives. In addition, the stratification of the parameters retrieved from the inversion imposes some restrictions that will prevent us from obtaining a physical solution because of the uncertainties in the determination of the magnetic field out of the optimal sensitivity region of the spectral lines. Accounting for all this complexity is a huge challenge and is beyond the scope of this paper (see Puschmann et al. 2010, for an example where the Lorentz force is included in the force balance equation). Finally, we obtained $\Delta z_{\mathrm{WD}}(x, y)$ by imposing pressure balance at one specific height. The balance is not satisfied at all $z$, and thus choosing a different height as a reference would lead to some differences in the Wilson depression.

Figure 12 illustrates the Wilson depression inferred for the observed sunspot. The maximum value of the Wilson depression is almost $600 \mathrm{~km}$. We obtain average values for $\Delta z_{\mathrm{WD}}(x, y)$ around $500 \mathrm{~km}$ for the dark umbra, $330 \mathrm{~km}$ for the faint umbra, $110 \mathrm{~km}$ for the penumbra, and $8 \mathrm{~km}$ for the light bridge. The light-bridge photosphere is basically at the height of the quietSun photosphere, and at some point, its Wilson depression is $-100 \mathrm{~km}$, that is, the surface at those locations is above the average surface of the quiet Sun. The contrast in $\Delta z_{\mathrm{WD}}(x, y)$ of the light bridge with respect to the dark surrounding regions is in the range $200-400 \mathrm{~km}$, in agreement with previous measurements (e.g., Lites et al. 2004).

\subsection{Three-dimensional structure}

The two-dimensional maps of the solar surface scanned during the observations and the depth-dependent stratification of the inferred physical parameters obtained with SIR allow us to construct a three-dimensional model of the observed active region. Figure 13 illustrates the thermodynamic and magnetic structure of the sunspot in the local reference frame after transforming the optical depth scale of the stratification into a geometrical scale taking into account the Wilson depression. All the parameters are plotted for $\log \tau$ lower than 0.3 , so that we are only including the region where the sensitivity of the spectral lines is high enough.
The top panel of Fig. 13 shows the region presented in Figs. 7-10, with the horizontal direction expressed in megameters and the position $(x=0, y=0)$ corresponding to the center of the umbra. The continuum intensity is plotted at the surface $\log \tau=0$. Magnetic field lines exhibit a radial geometry. Their color scale illustrates the magnetic field strength at the corresponding location. At the dark umbra magnetic field lines are mostly vertical and their strength is above $2000 \mathrm{G}$ (red). As farther regions from the center of the sunspot are considered, the magnetic field strength is reduced and its inclination is increased until it reaches the penumbra, where comparatively horizontal magnetic field lines dominate. In this panel, the field lines are plotted only where the magnetic field strength is above $500 \mathrm{G}$.

For a more detailed analysis of the light bridge, the middle panel of Fig. 13 shows an expanded plot of the domain delimited by the light blue box in the top panel. The point of view of the middle picture is indicated by the eye in the top panel. In this plot, the $z$-scale has been increased by a factor of 3 to better illustrate the Wilson depression. Some selected field lines that cross the light bridge have been plotted to illustrate the magnetic field configuration. The center of the umbra is pushed down almost $600 \mathrm{~km}$ with respect to the quiet-Sun surface. The light bridge shows a remarkable variation of the height at which the continuum intensity is formed, since it comes from a layer between 200 and $400 \mathrm{~km}$ higher than the $\log \tau=0$ height at either side. A movie showing the three-dimensional structure of the light bridge is available in the online material.

Figure 14 illustrates the plasma- $\beta$ at a surface with constant optical depth $\log \tau=0$ on a $\operatorname{logarithmic}$ scale. In the umbra (dark and faint), the isosurface $\log \tau=0$ lies above the $\beta=1$ surface, indicating that the continuum is formed in a low- $\beta$ regime, where the magnetic pressure is higher than the gas pressure. The photospheric umbra is dominated by a magnetic field with plasma- $\beta$ values as low as 0.2 . At the penumbra the photosphere changes from a magnetic-dominated regime at the umbra-penumbra boundary to a plasma-dominated regime at the boundary of the penumbra to the quiet-Sun. Strong variations in the plasma- $\beta$ associated with penumbral filaments are found west of the penumbra. On the one hand, bright penumbral filaments exhibit a plasma- $\beta$ roughly between 10 and 1000 . On the other hand, the plasma- $\beta$ in dark penumbral regions is around unity. The light bridge shows a striking increase in the plasma- $\beta$. The small umbra east of the light bridge is dominated by magnetic pressure, with a plasma- $\beta$ around 0.2 . However, in the light bridge the plasma- $\beta$ is in the range $1-200$.

The dynamics of the light bridge are clearly dominated by plasma motions, and this fact has a critical relevance for reshaping the magnetic field. The aforementioned velocity flows, consisting of a $1 \mathrm{~km} \mathrm{~s}^{-1}$ upflow surrounded by two downflows with a magnitude of $2 \mathrm{~km} \mathrm{~s}^{-1}$ at $\log \tau=0.3$ and decreasing with height, indicates that the light bridge exhibits vigorous convection. Since in the light bridge the magnetic field is frozen to the plasma motion, magnetic field lines are bent and become more horizontal just on top of the $\log \tau=0$ surface. At some locations they are even completely reversed, exhibiting a change in the polarity of the magnetic field. The bending of the field lines produced by the convective motions is clearly visible at the purple section of the field lines plotted in the middle panel of Fig. 13.

The small umbra east of the light bridge imposes a strong almost vertical magnetic field (red vertical field lines in the middle panel of Fig. 13). When the field lines crossing the light bridge reach those originating at the small umbra, the magnetic field tension is strong enough to reverse again the direction of the field lines. At those locations, they point in the same direction as 

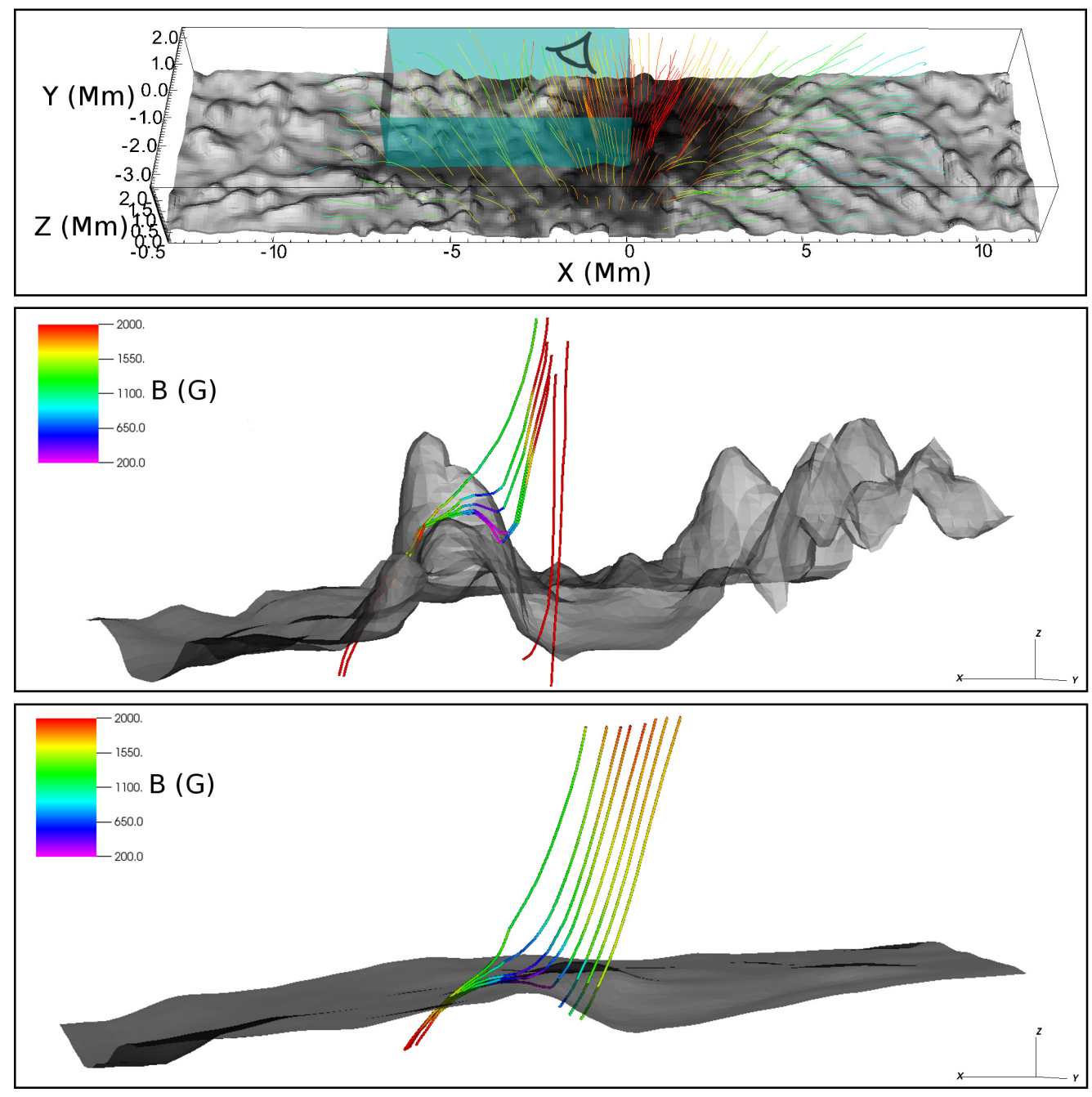

Fig. 13. Three-dimensional structure of the sunspot in the local reference frame. Continuum intensity is plotted at constant $\log \tau=0$ in gray scale. Magnetic field lines are illustrated with a color scale showing the magnetic field strength at the corresponding location from lower than $200 \mathrm{G}$ (purple) to higher than $2000 \mathrm{G}$ (red). Middle panel: detailed view of the light bridge region (delimited by the light blue box in the top panel and from the point of view indicate by the eye in top panel) and some selected field lines obtained from the analysis of the deconvolved maps (see Sect. 2.1). Bottom panel: detailed view of the light bridge region (same region as illustrated in the middle panel) and some selected field lines obtained from the analysis of the original non-deconvolved maps (see Sect. 2.1). In the middle and bottom panels the $z$-axis has been expanded by a factor three. The three-dimensional structure is illustrated as a movie in the online edition. In that movie the arrows indicate the velocity flows.

they did before crossing the light bridge. The field lines return to a close to vertical configuration, joining at some height the magnetic field lines from the other side of the light bridge (middle panel of Fig. 13).

Across the light bridge the magnetic field strength is significantly reduced. The variation of the magnetic field (in comparison with the surrounding dark umbra) decreases with height and extends from around $\Delta B_{\mathrm{ch}}=2200 \mathrm{G}$ at $z=0$ to $\Delta B_{\mathrm{ch}}=1000 \mathrm{G}$ at $z=1 \mathrm{Mm}$. As pointed out before, the magnetic field lines form a canopy. Below this canopy the magnetic field strength is even lower.

\subsection{Original vs. deconvolved maps}

The analysis of the sunspot was repeated using the original maps instead of those obtained from the deconvolution discussed in Sect. 2.1. The deconvolution allowed us to take into account the PSF of the telescope (including the effect of seeing) and correct for the stray light contamination.

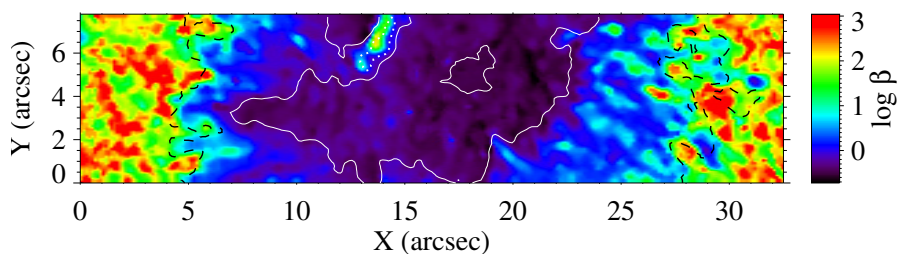

Fig. 14. Plasma- $\beta$ at $\log \tau=0$ in logarithmic scale. Contour lines correspond to the masks defined in Fig. 3 .

In both cases we obtain a similar global structure. The umbral and penumbral magnetic field show a radial structure. Regarding the light bridge, both cases point to a cusp-like magnetic field configuration, with a lower magnetic field below the canopy and a more vertical field at higher layers. However, there are also significant differences. The canopy obtained from the original maps is formed by mainly horizontal magnetic fields. Some reversals in the field polarization are also measured, and small deviations of the field lines from the horizontal are found. 
However, their bending is barely visible in the bottom panel of Fig. 13, which shows the results obtained from the analysis of the original data for the same region as is plotted in the middle panel of that figure. In contrast, the deconvolved map shows clear signatures of the twisting of the field lines produced by convective motions.

These light bridge convective motions, clearly visible in the velocity map retrieved from the analysis of the deconvolved map, are absent from the flow inferred from the original map. The latter only exhibits a $2 \mathrm{~km} \mathrm{~s}^{-1}$ downflow at the side of the light bridge next to the small umbra.

Our estimation of the Wilson depression in the deconvolved map (around $600 \mathrm{~km}$ in the umbra) is higher than that obtained by Mathew et al. (2004) using a similar approach (around $400 \mathrm{~km}$ ) and agrees with that retrieved from geometric techniques using the Wilson effect $(600 \pm 200 \mathrm{~km}$ Gokhale \& Zwaan 1972). Mathew et al. (2004) considered that the difference in their derived Wilson depression is due to neglecting the magnetic tension. However, from the analysis of the original map, the maximum Wilson depression that we obtain in the umbra is slightly higher than $300 \mathrm{~km}$. This result is closer to the value given by Mathew et al. (2004) and significantly lower that the value inferred with a better resolved map.

The magnitude of the Wilson depression inferred from the original data is strikingly lower than that from the deconvolved map. This effect is specially evident at the light bridge. The height of the $\log \tau=0$ surface around the light bridge in the deconvolved and original maps is illustrated in the middle and bottom panels of Fig. 13, respectively. The height difference between the low field strength regions and their surroundings retrieved from the analysis of the original map is below $100 \mathrm{~km}$, while in the deconvolved map this jump is up to $400 \mathrm{~km}$, in agreement with Lites et al. (2004).

\section{Discussion and conclusions}

We have analyzed the magnetic topology and thermodynamic structure of parts of a sunspot in active region NOAA 12096 a few days before it completely decayed. Our analysis has been focused on the photosphere, with special attention to the magnetic configuration of the deep photosphere that can be explored thanks to the Ca I $10839 \AA$ line. This spectral line is sensitive to deeper layers than other commonly used photospheric lines.

The inversion of the active region reveals many well-known and widely explored sunspot properties. Among other features, we showed the temperature structure and stratification of the sunspot atmosphere, traces of the Evershed flow, changes in the magnetic field from strong and vertical in the umbra to weaker and mostly horizontal in the penumbra, and the Wilson depression throughout the active region. The most compelling feature for further exploration is the deep photosphere in the light bridge that was formed between the main umbra and a nearby small umbral region. The magnetic field at this location is not only much lower than in the surrounding umbral regions, but also lower than the penumbral magnetic field. At the same time, its inclination with respect to the local vertical is also lower, showing horizontal field lines and even reversals in their trajectory. The vertical stratification of the light bridge atmosphere is clearly different from other penumbral regions.

The magnetic field structure of the light bridge reveals several similarities with the configuration that has been found for previously analyzed light bridges: it exhibits a lower and more horizontal magnetic field relative to the umbra (Beckers \& Schröter 1969; Lites et al. 1991; Leka 1997), its brightness is similar to the penumbra, and it is separated into two regions with the same polarity (Sobotka et al. 1993; Rimmele 2008), the higher layers are comparable to the surrounding umbral atmosphere (Rüedi et al. 1995), and the field lines form a cusp-like structure with a reduced field region in the inner part (Leka 1997; Jurčák et al. 2006; Lagg et al. 2014). In addition, the velocity profile shows central hot upflows surrounded by cooler downflows. This flow pattern has been detected in other light bridges, and it is interpreted as a evidence for vigorous convection in the light bridge (Rouppe van der Voort et al. 2010; Lagg et al. 2014).

The flow pattern measured for the light bridge exhibits some differences with respect to those observed in previous analyses. In this light bridge we do not detect a complete convective cell, where the upflow is completely surrounded by downflows. Instead, we only detected downflows at the side of the light bridge closer to the small umbra. The width of this cell $\left(1.9^{\prime \prime}\right)$ is comparable to the typical width of quiet-Sun granules and those observed in light bridges (1-2" Lagg et al. 2014). Only one of these convective cells is found in the analyzed light bridge, whose convective motions resemble those of quiet-Sun granules, except for the asymmetry in its downflows. The light bridge is relatively small, and it is possible that missing convective downflows are spatially unresolved near the umbral edges of both sides of the light bridge. Pressure and horizontal motions toward the edges of the light bridge could squeeze these downflows to very small spatial scales that are beyond the reach of the spatial resolution achieved even with the deconvolution described in Sect. 2.1, since the small-scale stray light could not be properly characterized. We also find some signatures of convection in the flow pattern at the center side of the penumbra (see the range $x=\left[25^{\prime \prime}, 28^{\prime \prime}\right]$ and $y=\left[6^{\prime \prime}, 8^{\prime \prime}\right]$ in the bottom panel of Fig. 8). Convective motions in the penumbra have been pointed out by previous observational works (Ichimoto et al. 2007; Zakharov et al. 2008; Joshi et al. 2011; Scharmer et al. 2011; Tiwari et al. 2013).

As discussed in Sect. 2.2, the light bridge is created by the motion of a small umbral region toward the main umbra (see movie). It is composed of the penumbral and quiet-Sun atmosphere that remained confined between two highly magnetized regions at the time the analyzed data were acquired. As the two magnetized regions approach each other, the field lines that spread from the central umbra are blocked by those coming from the dark pore-like feature. The field lines above the light bridge are forced to become more vertical than in the rest of the penumbra (see bottom panel of Fig. 11 around $\log \tau=-2$ ). This process forms a magnetic canopy. An atmosphere with a very low magnetic field and quiet-Sun temperature (see top and middle panel of Fig. 11) remains covered by a cusp-like field configuration. Convection continues, but the flows are confined by the low- $\beta$ atmosphere of the surrounding umbrae. Below the canopy, the gas pressure is much higher than the magnetic pressure (plasma- $\beta$ in the range 10-200). The downflowing material due to convection can drag the interior the magnetic field down below the canopy, bending the field lines and causing reversals in the field orientation. Closer to the small umbra, the magnetic tension can reverse the field lines again, which recover an almost vertical configuration and join the vertical field lines from the other side of the umbra. The magnetic canopy confines the plasma to the lower atmosphere. An example of this phenomena was recently found by Louis et al. (2015), who reported the emergence of a small loop in the lower atmosphere of a sunspot light bridge. The loop does not reach the chromosphere since the magnetic field configuration prevents it from continuing to rise. 
Field line reversals in light bridges were recently detected by Lagg et al. (2014). The authors proposed two possible scenarios: the field lines reverse their direction again due to magnetic tension, or the field is dragged toward the interior and the field lines are then directed downward. Our results have confirmed the bending in the field lines and have shown evidence that the first scenario of Lagg and collaborators is the most plausible. The detection of the two successive reversals in the field lines is only possible thanks to the high sensitivity of the Ca I $10839 \AA$ line to the deep layers and the high spatial resolution of the GREGOR telescope. Many works have reported chromospheric activity phenomena above light bridges (e.g., Roy 1973; Asai et al. 2001; Berger \& Berdyugina 2003; Shimizu et al. 2009; Toriumi et al. 2015; Robustini et al. 2016). Our observations in He I $10830 \AA$ do not show any trace of these dynamic events. However, the magnetic configuration found in our work at the deep photosphere can trigger these phenomena through reconnection of the reversed field lines.

The inversions carried out in this paper were performed independently using the original map and the spatially deconvolved map, which takes into account the telescope and seeing PSF and the stray light contamination. Both results show a similar general picture. However, the inversion of the original map also presents significant differences with the analysis of the deconvolved map. First, it does not reveal a clear pattern of the overturning convective motions of the light bridge. Instead, only a downflow is obtained. Second, the complex bending of the field lines in the light bridge is not unambiguously detected. Opposite-polarity fields are also found in the original non-deconvolved maps, but the curvature of the field lines is much lower. Finally, the magnitude of the Wilson depression is greatly underestimated. Our results indicate the remarkable relevance of correcting for telescope resolution and stray light to infer the magnetic and thermodynamic structure of the Sun from spectropolarimetric observations.

The decay of a sunspot is a long process during which the structure of the atmosphere shows a striking and complex evolution. Our analysis revealed the magnetic field configuration of the lower photosphere at a specific time close to the final stages of a sunspot. A comprehensive study of the magnetic configuration during the decay will require tracking an active region over several days. This study would also benefit from the inclusion of many active regions, sampling each stage of the evolution at different positions on the solar disk to analyze the magnetic and thermodynamic configuration under different viewing geometries.

Acknowledgements. The authors thank Basilio Ruiz Cobo and Carlos Quintero Noda for advice on inverting the data and providing very useful suggestions. The 1.5-m GREGOR solar telescope was built by a German consortium under the leadership of the Kiepenheuer-Institut für Sonnenphysik in Freiburg with the Leibniz-Institut für Astrophysik Potsdam, the Institut für Astrophysik Göttingen, and the Max-Planck-Institut für Sonnensystemforschung in Göttingen as partners, and with contributions by the Instituto de Astrofísica de Canarias and the Astronomical Institute of the Academy of Sciences of the Czech Republic. SDO HMI data are provided by the Joint Science Operations Center - Science Data Processing. The support of the European Commission's FP7 Capacities Programme under Grant Agreement number 312495 "SOLARNET" is acknowledged. This research has been funded by the Spanish MINECO through grant AYA2014-55078-P.

\section{References}

Asai, A., Ishii, T. T., \& Kurokawa, H. 2001, ApJ, 555, L65

Asplund, M., Grevesse, N., Sauval, A. J., \& Scott, P. 2009, ARA\&A, 47, 481

Balthasar, H., \& Collados, M. 2005, A\&A, 429, 705

Balthasar, H., \& Gömöry, P. 2008, A\&A, 488, 1085
Balthasar, H., Gömöry, P., González Manrique, S. J., et al. 2016, ArXiv e-prints [arXiv: 1609.01514]

Beck, C. 2008, A\&A, 480, 825

Beck, C., Rezaei, R., \& Fabbian, D. 2011, A\&A, 535, A129

Beckers, J. M., \& Schröter, E. H. 1969, Sol. Phys., 10, 384

Bello González, N., Okunev, O. V., Domínguez Cerdeña, I., Kneer, F., \& Puschmann, K. G. 2005, A\&A, 434, 317

Bellot Rubio, L. R., Tsuneta, S., Ichimoto, K., et al. 2007, ApJ, 668, L91

Bellot Rubio, L. R., Tritschler, A., \& Martínez Pillet, V. 2008, ApJ, 676, 698

Berger, T. E., \& Berdyugina, S. V. 2003, ApJ, 589, L117

Berkefeld, T., Schmidt, D., Soltau, D., von der Lühe, O., \& Heidecke, F. 2012, Astron. Nachr., 333, 863

Bloomfield, D. S., Solanki, S. K., Lagg, A., Borrero, J. M., \& Cally, P. S. 2007, A\&A, 469, 1155

Borrero, J. M., \& Ichimoto, K. 2011, Liv. Rev. Sol. Phys., 8, 4

Borrero, J. M., Bellot Rubio, L. R., Barklem, P. S., \& del Toro Iniesta, J. C. 2003, A\&A, 404, 749

Bray, R. J., \& Loughhead, R. E. 1964, Sunspots (London: Chapman \& Hall)

Centeno, R., Collados, M., \& Trujillo Bueno, J. 2006, ApJ, 640, 1153

Chae, J., Yun, H. S., Sakurai, T., \& Ichimoto, K. 1998, Sol. Phys., 183, 229

Collados, M. 1999, in Third Advances in Solar Physics Euroconference:

Magnetic Fields and Oscillations, eds. B. Schmieder, A. Hofmann, \& J. Staude, ASP Conf. Ser., 184, 3

Collados, M., López, R., Páez, E., et al. 2012, Astron. Nachr., 333, 872

Collados, M., Martinez Pillet, V., Ruiz Cobo, B., del Toro Iniesta, J. C., \& Vazquez, M. 1994, A\&A, 291, 622

Collados, M. V. 2003, in Polarimetry in Astronomy, ed. S. Fineschi, Proc. SPIE, 4843, 55

del Toro Iniesta, J. C., Tarbell, T. D., \& Ruiz Cobo, B. 1994, ApJ, 436, 400

Deng, N., Choudhary, D. P., Tritschler, A., et al. 2007, ApJ, 671, 1013

Evershed, J. 1909, MNRAS, 69, 454

Felipe, T., Khomenko, E., Collados, M., \& Beck, C. 2010, Apj, 722, 131

Garcia de La Rosa, J. I. 1987, Sol. Phys., 112, 49

Gary, G. A. 2001, Sol. Phys., 203, 71

Gingerich, O., Noyes, R. W., Kalkofen, W., \& Cuny, Y. 1971, Sol. Phys., 18, 347 Gokhale, M. H., \& Zwaan, C. 1972, Sol. Phys., 26, 52

Harvey, K., \& Harvey, J. 1973, Sol. Phys., 28, 61

Henriques, V. M. J. 2012, A\&A, 548, A114

Hirzberger, J., Bonet, J. A., Sobotka, M., Vázquez, M., \& Hanslmeier, A. 2002, A\&A, 383, 275

Hofmann, A., Arlt, K., Balthasar, H., et al. 2012, Astron. Nachr., 333, 854

Ichimoto, K., Suematsu, Y., Tsuneta, S., et al. 2007, Science, 318, 1597

Joshi, J., Lagg, A., Solanki, S. K., et al. 2016, A\&A, 596, A8 (GREGOR SI)

Joshi, J., Pietarila, A., Hirzberger, J., et al. 2011, ApJ, 734, L18

Jurčák, J., Martínez Pillet, V., \& Sobotka, M. 2006, A\&A, 453, 1079

Kuckein, C., Martínez Pillet, V., \& Centeno, R. 2012a, A\&A, 539, A131

Kuckein, C., Martínez Pillet, V., \& Centeno, R. 2012b, A\&A, 542, A112

Kurucz, R. L., Furenlid, I., Brault, J., \& Testerman, L. 1984, Solar Flux Atlas from 296 to $1300 \mathrm{~nm}$ (New Mexico: National Solar University)

Lagg, A., Solanki, S. K., van Noort, M., \& Danilovic, S. 2014, A\&A, 568, A60 Langhans, K., Scharmer, G. B., Kiselman, D., Löfdahl, M. G., \& Berger, T. E. 2005, A\&A, 436, 1087

Leka, K. D. 1997, ApJ, 484, 900

Leka, K. D., Barnes, G., Crouch, A. D., et al. 2009, Sol. Phys., 260, 83

Lites, B. W., Bida, T. A., Johannesson, A., \& Scharmer, G. B. 1991, ApJ, 373, 683

Lites, B. W., Elmore, D. F., Seagraves, P., \& Skumanich, A. P. 1993, ApJ, 418, 928

Lites, B. W., Low, B. C., Martinez Pillet, V., et al. 1995, ApJ, 446, 877

Lites, B. W., Scharmer, G. B., Berger, T. E., \& Title, A. M. 2004, Sol. Phys., 221, 65

Löhner-Böttcher, J., \& Schlichenmaier, R. 2013, A\&A, 551, A105

Louis, R. E., Bellot Rubio, L. R., de la Cruz Rodríguez, J., Socas-Navarro, H., \& Ortiz, A. 2015, A\&A, 584, A1

Martinez Pillet, V. 1992, Sol. Phys., 140, 207

Martínez Pillet, V. 2002, Astron. Nachr., 323, 342

Martinez Pillet, V., \& Vazquez, M. 1993, A\&A, 270, 494

Martínez Pillet, V., Lites, B. W., \& Skumanich, A. 1997, ApJ, 474, 810

Mathew, S. K., Solanki, S. K., Lagg, A., et al. 2004, A\&A, 422, 693

Meyer, F., Schmidt, H. U., Wilson, P. R., \& Weiss, N. O. 1974, MNRAS, 169, 35

Puschmann, K. G., Ruiz Cobo, B., \& Martínez Pillet, V. 2010, ApJ, 720, 1417

Quintero Noda, C., Asensio Ramos, A., Orozco Suárez, D., \& Ruiz Cobo, B.

2015, A\&A, 579, A3

Rimmele, T. 2008, ApJ, 672, 684

Rimmele, T. R. 1997, ApJ, 490, 458

Robustini, C., Leenaarts, J., de la Cruz Rodriguez, J., \& Rouppe van der Voort, L. 2016, A\&A, 590, A57 
T. Felipe et al.: Three-dimensional structure of a sunspot light bridge

Rouppe van der Voort, L., Bellot Rubio, L. R., \& Ortiz, A. 2010, ApJ, 718, L78 Roy, J. R. 1973, Sol. Phys., 28, 95

Rüedi, I., Solanki, S. K., \& Livingston, W. C. 1995, A\&A, 293, 252

Ruiz Cobo, B., \& del Toro Iniesta, J. C. 1992, 398, 375

Ruiz Cobo, B., \& Asensio Ramos, A. 2013, A\&A, 549, L4

Sánchez Almeida, J. 2005, ApJ, 622, 1292

Scharmer, G. B., Henriques, V. M. J., Kiselman, D., \& de la Cruz Rodríguez, J. 2011, Science, 333, 316

Schlichenmaier, R., \& Franz, M. 2013, A\&A, 555, A84

Schlichenmaier, R., von der Lühe, O., Hoch, S., et al. 2016, A\&A, 596, A7 (GREGOR SI)

Schmidt, W., von der Lühe, O., Volkmer, R., et al. 2012, Astron. Nachr., 333, 796

Schou, J., Scherrer, P. H., Bush, R. I., et al. 2012, Sol. Phys., 275, 229

Sheeley, Jr., N. R. 1972, Sol. Phys., 25, 98

Shimizu, T., Katsukawa, Y., Kubo, M., et al. 2009, ApJ, 696, L66

Sobotka, M., Bonet, J. A., \& Vazquez, M. 1993, ApJ, 415, 832

Sobotka, M., Bonet, J. A., \& Vazquez, M. 1994, ApJ, 426, 404

Solanki, S. K. 2003, A\&ARv, 11, 153
Solanki, S. K., Walther, U., \& Livingston, W. 1993, A\&A, 277, 639 Stanchfield, II, D. C. H., Thomas, J. H., \& Lites, B. W. 1997, ApJ, 477, 485 Tiwari, S. K., van Noort, M., Lagg, A., \& Solanki, S. K. 2013, A\&A, 557, A25

Tiwari, S. K., van Noort, M., Solanki, S. K., \& Lagg, A. 2015, A\&A, 583, A119 Toriumi, S., Katsukawa, Y., \& Cheung, M. C. M. 2015, ApJ, 811, 137

van Noort, M., Rouppe van der Voort, L., \& Löfdahl, M. G. 2005, Sol. Phys., 228,191

Vazquez, M. 1973, Sol. Phys., 31, 377

Verma, M., Balthasar, H., Deng, N., et al. 2012, A\&A, 538, A109

Volkmer, R., Eisenträger, P., Emde, P., et al. 2012, Astron. Nachr., 333, 816

von der Luehe, O. 1993, A\&A, 268, 374

Wedemeyer-Böhm, S. 2008, A\&A, 487, 399

Westendorp Plaza, C., del Toro Iniesta, J. C., Ruiz Cobo, B., et al. 2001, ApJ, 547,1130

Zakharov, V., Hirzberger, J., Riethmüller, T. L., Solanki, S. K., \& Kobel, P. 2008, A\&A, 488, L17

Zhang, H., Ai, G., Wang, H., Zirin, H., \& Patterson, A. 1992, Sol. Phys., 140, 307 